\title{
Test particle in a quantum gas
}

\author{
Bassano Vacchini* \\ Dipartimento di Fisica dell'Università di Milano and Istituto Nazionale di Fisica Nucleare, \\ Sezione di Milano, Via Celoria 16, I-20133, Milan, Italy
}

(Received 9 June 2000; published 22 May 2001)

\begin{abstract}
A master equation with a Lindblad structure is derived, which describes the interaction of a test particle with a macroscopic system and is expressed in terms of the operator valued dynamic structure factor of the system. In the case of a free Fermi or Bose gas the result is evaluated in the Brownian limit, thus obtaining a single generator master equation for the description of quantum Brownian motion in which the correction due to quantum statistics is explicitly calculated. The friction coefficients for Boltzmann and Bose or Fermi statistics are compared.
\end{abstract}

DOI: 10.1103/PhysRevE.63.066115

PACS number(s): 05.40.Jc, 05.30.Fk, 05.30.Jp, 05.60.Gg

\section{INTRODUCTION}

The study of the dynamics of a particle coupled to a general many-body system plays a relevant role in modern quantum physics, both with respect to foundations and applications of quantum theory. On the one hand it provides a most simple example of quantum dynamics of a nonisolated system, possibly offering a manageable arena for a truly microscopic approach, which might shed some light on mechanisms of dissipation and decoherence [1]; these last two issues are now of outstanding relevance in connection with the rapidly growing experimental ability to deal with thoroughly quantum-mechanical phenomena, checking for their coherence properties (at the single-particle level think, for example, of the recent cavity QED and ion trapping experiments [2], while at the many-body level, Bose-Einstein condensation is a most interesting example [3]). On the other hand, plenty of interesting physical problems may be modeled in this way and among these, in particular, motion or diffusion of charged or neutral particles in gases, liquids, or solids. The interaction of a test particle with a dilute or noninteracting gas is strictly connected to the problem of a quantum generalization of the Boltzmann equation [4], whose everlasting relevance has recently been stressed by the experimental realization of quantum degenerate samples of weakly interacting bosons or fermions $[3,5]$; in fact for the study of these systems, resort is often made to a quantum Boltzmann transport equation [6]. A particularly interesting situation arises if the mass $M$ of the test particle is much bigger than the mass $m$ of the particles, which make up the gas; the so-called Brownian motion, which serves as a paradigmatic example in the description of irreversible and dissipative processes. The description of the phenomenon is still debated at a quantum level (see Refs. [7,8] and references cited therein), even though well settled by now at the classical level in terms of Langevin or Fokker-Planck equations (it took however almost a century from the observation by Brown to the first successful theoretical description by Einstein, which led to the first example of a fluctuation-

*Email address: bassano.vacchini@mi.infn.it dissipation relation, linking friction and diffusion coefficients). A class of models, usually named quantum Brownian motion [9], given by time evolutions with a non-Hamiltonian part mapping the algebra of operators at most bilinear in the operators $\hat{\boldsymbol{x}}$ and $\hat{\boldsymbol{p}}$ of the particle into itself, seem to be the most natural candidate in order to obtain equations of motion analogous to the classical ones, leading in particular to a friction force proportional to velocity. On the mathematical side, generators of time evolution semigroups satisfying these requirements have been fully characterized through the property of complete positivity, which formally amounts to the requirement that positivity of the time evolution is preserved even in the presence of coupling without interaction to another system and leads to a typical expression for the generators of these semigroups, known as the Lindblad structure [10]. This has led to a wide literature developing this axiomatic approach [11], together with a large number of more or less phenomenological models in which similar structures are obtained, though not always preserving complete positivity (in this connection see Ref. [12]). Though warranting positivity of the statistical operator, complete positivity is by itself no fundamental requirement as recently stressed [13], so that despite its extensive use in many fields of physics, ranging from quantum optics to quantum communication, the study of the conditions and approximations under which it emerges from microphysical models is strongly desirable.

In a recent work, the derivation at a fundamental level of a completely positive master equation for a Brownian particle interacting with a free Boltzmann gas has been given, based on a microphysical model developed for the description of particle matter interaction [14,7]. The Lindblad equation thus obtained can be written with a single generator and temperature-dependent friction and diffusion coefficients were determined in terms of the scattering cross section. In this paper we give a major extension of the previous model, keeping also quantum statistics of the gas into account. Moreover, before going over to the Brownian limit, in which the ratio between the masses is much smaller than one, one sees that the generator of the master equation is expressed in terms of the dynamic structure factor of the medium, first introduced by van Hove [15]. This turns out to be true also 
for an interacting system, thus linking in full generality the dynamics of the test particle to the density fluctuations of the system [see Eq. (20)]. The property of complete positivity is retained in the general case under some requirements on the energy dependence of the dynamic structure factor, which are exactly fulfilled in the case of Boltzmann particles dealt with in Ref. [7]. The Brownian limit is then considered, thus obtaining the correction at finite temperature due to quantum statistics to the equation describing quantum Brownian motion [see Eq. (27)]. In terms of the fugacity $z$ this correction takes a remarkably simple form [see Eq. (29)].

The paper is organized as follows: in Sec. II we consider the general structure of the master equation and its connection to the dynamic structure factor. In Sec. III we obtain the correction due to quantum statistics to the master equation describing quantum Brownian motion, together with the relationship between the friction coefficients for Boltzmann or quantum statistics. In Sec. IV we comment on our results indicating potential future developments.

\section{GENERAL STRUCTURE OF THE MASTER EQUATION IN TERMS OF THE DYNAMIC STRUCTURE FACTOR}

Let us briefly recall the structure of the master equation obtained in Ref. [14] for the description of the subdynamics of a particle interacting with a macroscopic system supposed to be at equilibrium. The result is valid on a time scale $\tau$ much longer than microphysical collision time and describes an interaction through two-particle collisions given by the full $\mathrm{T}$ matrix. The master equation is given by

$$
\frac{d \hat{\varrho}}{d t}=-\frac{i}{\hbar}\left[\hat{H}_{0}, \hat{\varrho}\right]+\frac{1}{\hbar} \sum_{\lambda, \xi}\left[\hat{L}_{\lambda \xi} \hat{\varrho} \hat{\varrho} \hat{L}_{\lambda \xi}^{\dagger}-\frac{1}{2}\left\{\hat{L}_{\lambda \xi}^{\dagger} \hat{L}_{\lambda \xi}, \hat{\varrho}\right\}\right],
$$

with

$$
\left\langle k\left|\hat{L}_{\lambda \xi}\right| h\right\rangle=\sqrt{2 \varepsilon \pi_{\xi}} \frac{\left\langle\lambda\left|T_{h}^{k}\right| \xi\right\rangle}{E_{k}+E_{\lambda}-E_{h}-E_{\xi}-i \varepsilon},
$$

where $\hat{H}_{0}$ is the Hamiltonian for the particle and $\hat{\varrho}$ its statistical operator, while $\varrho^{\mathrm{m}}=\Sigma_{\xi} \pi_{\xi}|\xi\rangle\langle\xi|$ is the statistical operator for matter at equilibrium, $\pi_{\xi}$ being the statistical weights related to its spectral decomposition. The vectors $|\lambda\rangle$ and $|\xi\rangle$ are eigenvectors of the macrosystem Hamiltonian $H_{\mathrm{m}}$ with eigenvalues $E_{\lambda}$ and $E_{\xi}$, respectively, similarly $|k\rangle$ and $|h\rangle$ denote eigenvectors of $\hat{H}_{0}$ with eigenvalues $E_{k}$, and $E_{h}$. In writing the equation we have neglected the slow energy dependence of the T matrix, which would have brought a commutator term proportional to the forward scattering amplitude, diagonal in momentum representation. The terms other than the commutator in Eq. (1) are linked to the dissipative behavior, which cannot be obtained in a Hamiltonian formalism. Interactions at the microphysical level are typically translationally invariant, so that a general ansatz for the $\mathrm{T}$ matrix describing two-body interactions is given by $T_{h}^{k}$ $=\int d^{3} \boldsymbol{x} \int d^{3} \boldsymbol{y} \psi^{\dagger}(\boldsymbol{x}) u_{k}^{*}(\boldsymbol{y}) t(\boldsymbol{x}-\boldsymbol{y}) u_{h}(\boldsymbol{y}) \psi(\boldsymbol{x})$, where $\psi^{\dagger}, \psi$ are field operators for the macrosystem. We now consider a ho- mogeneous system, so as to use as quantum numbers momentum eigenvalues, thus obtaining with a Fourier transform, an expression depending only on the modulus of the momentum transfer:

$$
T_{h}^{k}=\sum_{\eta \mu} \delta_{p_{\eta}+p_{k}, p_{h}+p_{\mu}} \tilde{t}\left(\left|\boldsymbol{p}_{\mu}-\boldsymbol{p}_{\eta}\right|\right) b_{\eta}^{\dagger} b_{\mu}
$$

where $b^{\dagger}, b$ denote creation and destruction operators in the Fock space of the macrosystem. Restricting to the case of a free gas of Bose or Fermi particles, the eigenvectors of $H_{\mathrm{m}}$ can be characterized as a set of occupation numbers $n_{\sigma}$ relative to particles with a given momentum $\boldsymbol{p}_{\sigma}$, so that $|\xi\rangle$ $=\left|\left\{n_{\sigma}^{\xi}\right\}\right\rangle$, and the matrix element $\left\langle\lambda\left|b_{\eta}^{\dagger} b_{\mu}\right| \xi\right\rangle$ can be readily evaluated restricted to the primed sum for $\lambda \neq \xi$, since in the case $\lambda=\xi$, the contributions to the master equation (1) cancel out. Denoting by $\boldsymbol{q}=\boldsymbol{p}_{\mu}-\boldsymbol{p}_{\eta}$, the momentum transferred to the test particle and by $\Delta E_{p_{\mu} q}(\boldsymbol{p})=(\boldsymbol{p}+\boldsymbol{q})^{2} / 2 M+\left(\boldsymbol{p}_{\mu}\right.$ $-\boldsymbol{q})^{2} / 2 m-\boldsymbol{p}^{2} / 2 M-\boldsymbol{p}_{\mu}^{2} / 2 m$ the difference in energy before and after the collision ( $M$ being the mass of the test particle with momentum $\boldsymbol{p}, m$ the mass of the gas particles), and supposing the statistical operator $\hat{\varrho}$ to be quasidiagonal in momentum representation, according to its slow variability, one sees that Eq. (1) for a free test particle reduces to [7]

$$
\begin{aligned}
\frac{d \hat{\varrho}}{d t}= & -\frac{i}{\hbar}\left[\frac{\hat{\boldsymbol{p}}^{2}}{2 M}, \hat{\varrho}\right]+\frac{2 \pi}{\hbar} \sum_{q}^{\prime}|\widetilde{t}(q)|^{2}\left\{\sum_{p p^{\prime}} \sum_{\mu}\left\langle n_{\mu}\right\rangle\right. \\
& \times\left(1 \pm\left\langle n_{\mu-q}\right\rangle\right) \delta\left[\Delta E_{p_{\mu} q}\left(\frac{\boldsymbol{p}+\boldsymbol{p}^{\prime}}{2}\right)\right] \\
& \times e^{(i / \hbar) \boldsymbol{q} \cdot \hat{x}}|\boldsymbol{p}\rangle\left\langle\boldsymbol{p}|\hat{\varrho}| \boldsymbol{p}^{\prime}\right\rangle\left\langle\boldsymbol{p}^{\prime}\right| e^{-(i / \hbar) \boldsymbol{q} \cdot \hat{\boldsymbol{x}}} \\
& -\frac{1}{2} \sum_{p} \sum_{\mu}\left\langle n_{\mu}\right\rangle\left(1 \pm\left\langle n_{\mu-q}\right\rangle\right) \\
& \left.\times \delta\left(\Delta E_{p_{\mu} q}(\boldsymbol{p})\right)\{|\boldsymbol{p}\rangle\langle\boldsymbol{p}|, \hat{\varrho}\}\right\},
\end{aligned}
$$

where the,+- signs refer to Bose, Fermi statistics, respectively, and

$$
\left\langle n_{\mu}\right\rangle=\frac{z e^{-\beta\left(p_{\mu}^{2} / 2 m\right)}}{1 \mp z e^{-\beta\left(p_{\mu}^{2} / 2 m\right)}}
$$

accordingly, $z$ denoting the fugacity, determined by the requirement $\Sigma_{\mu}\left\langle n_{\mu}\right\rangle=N$, and $\beta=1 /\left(k_{\mathrm{B}} T\right)$ the inverse temperature. It is worthwhile introducing the more compact notation

$$
S_{\mathrm{B} / \mathrm{F}}(\boldsymbol{q}, \boldsymbol{p})=\frac{1}{n} \int \frac{d^{3} \boldsymbol{p}_{\mu}}{(2 \pi \hbar)^{3}}\left\langle n_{p_{\mu}}\right\rangle\left(1 \pm\left\langle n_{p_{\mu}-q}\right\rangle\right) \delta\left(\Delta E_{p_{\mu} q}(\boldsymbol{p})\right),
$$


where $n$ denotes the density of particles in the gas and the function $S_{\mathrm{B} / \mathrm{F}}$ is in fact positive definite, so that Eq. (3) becomes

$$
\begin{aligned}
\frac{d \hat{\varrho}}{d t}= & -\frac{i}{\hbar}\left[\frac{\hat{\boldsymbol{p}}^{2}}{2 M}, \hat{\varrho}\right]+\frac{2 \pi}{\hbar}(2 \pi \hbar)^{3} n \int d^{3} \boldsymbol{q}|\tilde{t}(q)|^{2} \\
& \times\left[\int d^{3} \boldsymbol{p} \int d^{3} \boldsymbol{p}^{\prime} S_{\mathrm{B} / \mathrm{F}}\left(\boldsymbol{q}, \frac{\boldsymbol{p}+\boldsymbol{p}^{\prime}}{2}\right) e^{(i / \hbar) \boldsymbol{q} \cdot \hat{\boldsymbol{x}}}|\boldsymbol{p}\rangle\left\langle\boldsymbol{p}|\hat{\varrho}| \boldsymbol{p}^{\prime}\right\rangle\right. \\
& \left.\times\left\langle\boldsymbol{p}^{\prime}\right| e^{-(i / \hbar) \boldsymbol{q} \cdot \hat{x}}-\frac{1}{2} \int d^{3} \boldsymbol{p} S_{\mathrm{B} / \mathrm{F}}(\boldsymbol{q}, \boldsymbol{p})\{|\boldsymbol{p}\rangle\langle\boldsymbol{p}|, \hat{\varrho}\}\right]
\end{aligned}
$$

The integral in Eq. (4) can be explicitly calculated both for bosons and fermions giving at finite temperature the result

$$
\begin{aligned}
S_{\mathrm{B} / \mathrm{F}}(\boldsymbol{q}, \boldsymbol{p})= & \mp \frac{1}{(2 \pi \hbar)^{3}} \frac{2 \pi m^{2}}{n \beta q} \\
& \times \frac{1}{1-\exp \left\{(\beta / 2 m)\left[2 \sigma(\boldsymbol{q}, \boldsymbol{p}) q-q^{2}\right]\right\}} \\
& \times \log \left[\frac{1 \mp z \exp \left[-(\beta / 2 m) \sigma^{2}(\boldsymbol{q}, \boldsymbol{p})\right]}{1 \mp z \exp \left[-(\beta / 2 m)[\sigma(\boldsymbol{q}, \boldsymbol{p})-q]^{2}\right]}\right]
\end{aligned}
$$

where $\sigma(\boldsymbol{q}, \boldsymbol{p})=(1 / 2 q)\left[(1+\alpha) q^{2}+2 \alpha(\boldsymbol{p} \cdot \boldsymbol{q})\right]$ is expressed in terms of the dimensionless variable $\alpha=m / M$, giving the ratio between the masses. Expression (6) is exactly the dynamic structure factor for a free Bose or Fermi gas at finite temperature, as one could realize from Eq. (4) [16] or from the equivalent expression in terms of momentum transfer $\boldsymbol{q}$ and energy transfer $E=q^{2} / 2 M+\boldsymbol{p} \cdot \boldsymbol{q} / M$ (note that we use as variables momentum and energy transferred to the test particle)

$$
\begin{aligned}
S_{\mathrm{B} / \mathrm{F}}(q, E)= & \mp \frac{1}{(2 \pi \hbar)^{3}} \frac{2 \pi m^{2}}{n \beta q} \frac{1}{1-e^{\beta E}} \\
& \times \log \left[\frac{1 \mp z \exp \left[-(\beta / 8 m)\left(2 m E+q^{2}\right)^{2} / q^{2}\right]}{1 \mp z \exp \left[-(\beta / 8 m)\left(2 m E-q^{2}\right)^{2} / q^{2}\right]}\right],
\end{aligned}
$$

where the dependence on the transferred momentum is, in this case, actually only through the modulus. In the following we will use, according to convenience, both notations $S(\boldsymbol{q}, \boldsymbol{p})$ and $S(\boldsymbol{q}, E)$, where $E \equiv \Delta E_{q}(\boldsymbol{p})=E_{p+q}-E_{p}=q^{2} / 2 M+\boldsymbol{p}$ $\cdot \boldsymbol{q} / M$ is the energy transfer. The dynamic structure factor is an important physical quantity of direct experimental access, essentially depending on the statistical properties of the macrosystem and the kinematics of the collision, appearing in the expression of the inelastic differential cross section for a particle interacting with a macroscopic sample. The relation between differential cross section and dynamic structure factor was first derived by van Hove in the case of neutron scattering [15] and for scattering from state $\boldsymbol{p}$ to state $\boldsymbol{p}^{\prime}=\boldsymbol{p}+\boldsymbol{q}$, is given per target particle, by

$$
\frac{d^{2} \sigma}{d \Omega_{p^{\prime}} d E_{p^{\prime}}}=(2 \pi \hbar)^{6}\left(\frac{M}{2 \pi \hbar^{2}}\right)^{2} \frac{p^{\prime}}{p}|\widetilde{t}(q)|^{2} S(\boldsymbol{q}, E) .
$$

The dynamic structure factor is expressed in the general case as the Fourier transform of the time-dependent paircorrelation function with respect to energy and momentum transfer, according to

$$
\begin{aligned}
S(\boldsymbol{q}, E)= & \frac{1}{2 \pi \hbar} \frac{1}{N} \int d t \int d^{3} \boldsymbol{x} \\
& \times \exp \left[\frac{i}{\hbar}(E t-\boldsymbol{q} \cdot \boldsymbol{x})\right] \int d^{3} \boldsymbol{y}\langle N(\boldsymbol{y}) N(\boldsymbol{x}+\boldsymbol{y}, t)\rangle,
\end{aligned}
$$

where $N(\boldsymbol{y})$ denotes the local particle density for the macroscopic system and $\langle\ldots\rangle$ the ensemble average. Alternatively the dynamic structure factor may be written in terms of the Fourier transform of the density operator $N(\boldsymbol{y})$, given by

$$
\rho_{q}=\int d^{3} \boldsymbol{y} e^{-(i / \hbar) \boldsymbol{q} \cdot \boldsymbol{y}} N(\boldsymbol{y})=\sum_{\mu} b_{\mu}^{\dagger} b_{\mu+q},
$$

thus obtaining

$$
S(\boldsymbol{q}, E)=\frac{1}{2 \pi \hbar} \frac{1}{N} \int d t e^{(i / \hbar) E t}\left\langle\rho_{q}^{\dagger} \rho_{q}(t)\right\rangle .
$$

Expression (10) through relation (7) allows a determination of the equilibrium fluctuations of the system in terms of scattering experiments [16] (think, for example, of the very interesting applications in the case of neutron scattering from different states and isotopes of helium [17]). Coming back to Eq. (6) we note that in the limit of very small fugacity $z$ $\ll 1$, one recovers the result for Maxwell Boltzmann particles

$$
S_{\mathrm{MB}}(\boldsymbol{q}, \boldsymbol{p})=\frac{1}{(2 \pi \hbar)^{3}} \frac{2 \pi m^{2}}{n \beta q} z \exp \left[-\frac{\beta}{2 m} \sigma^{2}(\boldsymbol{q}, \boldsymbol{p})\right],
$$

which in terms of momentum and energy transfer may also be written

$$
S_{\mathrm{MB}}(q, E)=\frac{1}{(2 \pi \hbar)^{3}} \frac{2 \pi m^{2}}{n \beta q} z \exp \left[-\frac{\beta}{8 m} \frac{\left(2 m E+q^{2}\right)^{2}}{q^{2}}\right] .
$$

Recalling expression (4) for the dynamic structure factor one immediately realizes that the master equation given in Eq. (3) can be written in terms of the dynamic structure factor and exactly exhibits a Lindblad structure provided the dynamic structure factor evaluated at the arithmetic mean of $\boldsymbol{p}$ and $\boldsymbol{p}^{\prime}$ equals the geometric mean of its values at the two points. This identity holds true without approximations in the case of expression (22) for a Boltzmann gas in the Brownian limit considered in Ref. [7]. In the general case this factor- 
ization relies on an approximation linked to the quasidiagonality of the statistical operator. Keeping the linear relation between $E$ and $\boldsymbol{p}$ into account, the approximation necessary in order to retain complete positivity can be most meaningfully written

$$
S\left(\boldsymbol{q}, \frac{E+E^{\prime}}{2}\right) \approx \sqrt{S(\boldsymbol{q}, E)} \sqrt{S\left(\boldsymbol{q}, E^{\prime}\right)}
$$

and will depend on the smoothness of the energy dependence of $S$ in the relevant energy region (note that the neglected terms are at least quadratic in the energy difference). Exploiting Eq. (12), Eq. (5) can be cast in the following Lindblad structure granting positivity of the time evolution

$$
\begin{aligned}
\frac{d \hat{\varrho}}{d t}= & -\frac{i}{\hbar}\left[\frac{\hat{\boldsymbol{p}}^{2}}{2 M}, \hat{\varrho}\right]+\frac{2 \pi}{\hbar}(2 \pi \hbar)^{3} n \int d^{3} \boldsymbol{q}|\tilde{t}(q)|^{2} \\
& \times\left[e^{(i / \hbar) \boldsymbol{q} \cdot \hat{\boldsymbol{x}}} \sqrt{S_{\mathrm{B} / \mathrm{F}}(\boldsymbol{q}, \hat{\boldsymbol{p}})} \hat{\varrho} \sqrt{S_{\mathrm{B} / \mathrm{F}}(\boldsymbol{q}, \hat{\boldsymbol{p}})} e^{-(i / \hbar) \boldsymbol{q} \cdot \hat{\boldsymbol{x}}}\right. \\
& \left.-\frac{1}{2}\left\{S_{\mathrm{B} / \mathrm{F}}(\boldsymbol{q}, \hat{\boldsymbol{p}}), \hat{\varrho}\right\}\right],
\end{aligned}
$$

which may be also written in a more manifest Lindblad form

$$
\begin{aligned}
\frac{d \hat{\varrho}}{d t}= & -\frac{i}{\hbar}\left[\hat{\boldsymbol{H}}_{0}, \hat{\varrho}\right]+\frac{2 \pi}{\hbar}(2 \pi \hbar)^{3} n \int d^{3} \boldsymbol{q}|\tilde{t}(q)|^{2} \\
& \times\left[L_{\mathrm{B} / \mathrm{F}}(\boldsymbol{q}, \hat{\boldsymbol{p}}, \hat{\boldsymbol{x}}) \hat{\varrho} L_{\mathrm{B} / \mathrm{F}}^{\dagger}(\boldsymbol{q}, \hat{\boldsymbol{p}}, \hat{\boldsymbol{x}})\right. \\
& \left.-\frac{1}{2}\left\{L_{\mathrm{B} / \mathrm{F}}^{\dagger}(\boldsymbol{q}, \hat{\boldsymbol{p}}, \hat{\boldsymbol{x}}) L_{\mathrm{B} / \mathrm{F}}(\boldsymbol{q}, \hat{\boldsymbol{p}}, \hat{\boldsymbol{x}}), \hat{\varrho}\right\}\right],
\end{aligned}
$$

introducing the following generator depending on the operators $\hat{\boldsymbol{x}}$ and $\hat{\boldsymbol{p}}$

$$
L_{\mathrm{B} / \mathrm{F}}(\boldsymbol{q}, \hat{\boldsymbol{p}}, \hat{\boldsymbol{x}})=e^{(i / \hbar) \boldsymbol{q} \cdot \hat{\boldsymbol{x}}} \sqrt{S_{\mathrm{B} / \mathrm{F}}(\boldsymbol{q}, \hat{\boldsymbol{p}})} .
$$

This is a remarkably simple result since $L_{\mathrm{B} / \mathrm{F}}$ only depends on the generator of translations in momentum space and the operator valued dynamic structure factor. Let us note that equation (13) or equivalently Eq. (14) is invariant under translation and rotation and in particular, a statistical operator of the canonical form $\hat{\varrho} \propto e^{-\beta\left(\hat{p}^{2} / 2 M\right)}$, is a stationary solution [18]. If instead of a free gas one considers a more general medium characterized by a dynamic structure factor $S(\boldsymbol{q}, \boldsymbol{p})$, provided the interaction between particle and medium still satisfies translation invariance as in Eq. (2) and an approximation of the form (12) holds, the master equation (1) still has the form (13) or equivalently (14) with $L(\boldsymbol{q}, \hat{\boldsymbol{p}}, \hat{\boldsymbol{x}})$ given by

$$
L(\boldsymbol{q}, \hat{\boldsymbol{p}}, \hat{\boldsymbol{x}})=e^{(i / \hbar) \boldsymbol{q} \cdot \hat{\boldsymbol{x}}} \sqrt{S(\boldsymbol{q}, \hat{\boldsymbol{p}})}
$$

and therefore retains a Lindblad structure. To prove this we go back to Eq. (1), which in the case of a homogeneous system using Eq. (2) can be written

$$
\frac{d \hat{\varrho}}{d t}=-\frac{i}{\hbar}\left[\hat{H}_{0}, \hat{\varrho}\right]+\mathcal{L}(\hat{\varrho})
$$

with

$$
\begin{aligned}
\mathcal{L}(\hat{\varrho})= & \frac{2 \varepsilon}{\hbar} \sum_{\lambda \xi} \sum_{k f} \sum_{h g}\left|\boldsymbol{p}_{f}\right\rangle \frac{\sum_{\eta \mu} \delta_{p_{\eta^{\prime}}+p_{f}, p_{k}+p_{\mu}} \tilde{t}\left(\left|\boldsymbol{p}_{\mu}-\boldsymbol{p}_{\eta}\right|\right)\left\langle\lambda\left|b_{\eta}^{\dagger} b_{\mu}\right| \xi\right\rangle}{E_{k}-E_{f}+E_{\xi}-E_{\lambda}+i \varepsilon} \\
& \times\left\langle\boldsymbol{p}_{k}|\hat{\varrho}| \boldsymbol{p}_{h}\right\rangle \pi_{\xi} \frac{\sum_{\eta^{\prime} \mu^{\prime}} \delta_{p_{\eta^{\prime}}+p_{g}, p_{h}+p_{\mu^{\prime}}} \tilde{t}^{*}\left(\left|\boldsymbol{p}_{\mu^{\prime}}-\boldsymbol{p}_{\eta^{\prime}}\right|\right)\left\langle\xi\left|b_{\mu^{\prime}}^{\dagger} b_{\eta^{\prime}}\right| \lambda\right\rangle}{E_{h}-E_{g}+E_{\xi}-E_{\lambda}-i \varepsilon} \\
& -\frac{\varepsilon}{\hbar} \sum_{\lambda \xi} \sum_{k} \sum_{f g}\left\{\left|\boldsymbol{p}_{f}\right\rangle\left\langle\boldsymbol{p}_{g}\right|, \hat{\varrho}\right\} \frac{\sum_{\eta \mu} \delta_{p_{\eta}+p_{k}, p_{g}+p_{\mu}} \tilde{t}\left(\left|\boldsymbol{p}_{\mu}-\boldsymbol{p}_{\eta}\right|\right)\left\langle\lambda\left|b_{\eta}^{\dagger} b_{\mu}\right| \xi\right\rangle}{E_{f}-E_{k}+E_{\xi}-E_{\lambda}-i \varepsilon} \\
& \times \pi_{\xi} \frac{\sum_{\eta^{\prime} \mu^{\prime}} \delta_{p_{\eta^{\prime}}+p_{k}, p_{f}+p_{\mu^{\prime}}} \tilde{t}^{*}\left(\left|\boldsymbol{p}_{\mu^{\prime}}-\boldsymbol{p}_{\eta^{\prime}}\right|\right)\left\langle\xi\left|b_{\mu^{\prime}}^{\dagger} b_{\eta^{\prime}}\right| \lambda\right\rangle}{E_{g}-E_{k}+E_{\xi^{\prime}}-E_{\lambda}+i \varepsilon} .
\end{aligned}
$$

We now introduce the momentum transfer $\boldsymbol{q}=\boldsymbol{p}_{\mu}-\boldsymbol{p}_{\eta}, \boldsymbol{q}^{\prime}=\boldsymbol{p}_{\mu^{\prime}}-\boldsymbol{p}_{\eta^{\prime}}$ and the Fourier transform $\boldsymbol{\rho}_{q}$ of the density operator given by Eq. (9), so that relabeling the indexes (17) becomes 


$$
\begin{aligned}
\mathcal{L}(\hat{\varrho})= & \frac{2 \varepsilon}{\hbar} \sum_{\lambda \xi} \sum_{p p^{\prime}} \sum_{q q^{\prime}}{ }^{\prime} \tilde{t}(q) \tilde{t}^{*}\left(q^{\prime}\right) e^{(i / \hbar) \boldsymbol{q} \cdot \hat{\boldsymbol{x}}}|\boldsymbol{p}\rangle\left\langle\boldsymbol{p}|\hat{\varrho}| \boldsymbol{p}^{\prime}\right\rangle\left\langle\boldsymbol{p}^{\prime}\right| e^{-(i / \hbar) \boldsymbol{q}^{\prime} \cdot \hat{x}} \frac{1}{E_{p}-E_{p+q}+E_{\xi}-E_{\lambda}+i \varepsilon} \\
& \times \frac{1}{E_{p^{\prime}}-E_{p^{\prime}+q^{\prime}}+E_{\xi}-E_{\lambda}-i \varepsilon}\left\langle\lambda\left|\rho_{q}\right| \xi\right\rangle \pi_{\xi}\left\langle\xi\left|\rho_{q^{\prime}}^{\dagger}\right| \lambda\right\rangle-\frac{\varepsilon}{\hbar} \sum_{\lambda \xi} \sum_{p} \sum_{q q^{\prime}}{ }^{\prime} \tilde{t}(q) \tilde{t}^{*}\left(q^{\prime}\right)\{|\boldsymbol{p}\rangle\langle\boldsymbol{p}|, \hat{\varrho}\} \\
& \times \frac{1}{E_{p}-E_{p+q}+E_{\xi}-E_{\lambda}-i \varepsilon} \frac{1}{E_{p}-E_{p+q^{\prime}}+E_{\xi}-E_{\lambda}+i \varepsilon}\left\langle\lambda\left|\rho_{q}\right| \xi\right\rangle \pi_{\xi}\left\langle\xi\left|\rho_{q^{\prime}}^{\dagger}\right| \lambda\right\rangle,
\end{aligned}
$$

where the primed sum over $\boldsymbol{q}$ and $\boldsymbol{q}^{\prime}$ means that the contribution for $\boldsymbol{q}=\boldsymbol{q}^{\prime}=\mathbf{0}$ is left out, since in this case the two terms cancel out. To proceed further, we express the denominators in terms of a Laplace transform, according to

$$
(a \pm i \varepsilon)^{-1}=\mp \frac{i}{\hbar} \int_{0}^{\infty} d \tau e^{ \pm(i / \hbar)(a \pm i \varepsilon) \tau},
$$

thus obtaining

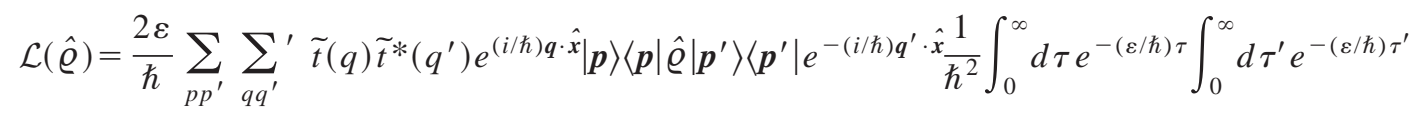

$$
\begin{aligned}
& \times \exp \left[-(i / \hbar) \Delta E_{q}(\boldsymbol{p}) \tau\right] \exp \left[+(i / \hbar) \Delta E_{q^{\prime}}\left(\boldsymbol{p}^{\prime}\right) \tau^{\prime}\right]\left\langle\rho_{q^{\prime}}^{\dagger} \rho_{q}\left(\tau-\tau^{\prime}\right)\right\rangle-\frac{\varepsilon}{\hbar} \sum_{p} \sum_{q q^{\prime}}^{\prime} \tilde{t}(q) \tilde{t}^{*}\left(q^{\prime}\right)\{|\boldsymbol{p}\rangle\langle\boldsymbol{p}|, \hat{\varrho}\} \\
& \times \frac{1}{\hbar^{2}} \int_{0}^{\infty} d \tau e^{-\frac{\varepsilon}{\hbar} \tau} \int_{0}^{\infty} d \tau^{\prime} e^{-(\varepsilon / \hbar) \tau^{\prime}} \exp \left[-(i / \hbar) \Delta E_{q}(\boldsymbol{p}) \tau\right] \exp \left[+(i / \hbar) \Delta E_{q^{\prime}}(\boldsymbol{p}) \tau^{\prime}\right]\left\langle\rho_{q^{\prime}}^{\dagger}, \rho_{q}\left(\tau-\tau^{\prime}\right)\right\rangle,
\end{aligned}
$$

where $\langle\ldots\rangle$ denotes the ensemble average over $\varrho^{\mathrm{m}}, \rho_{q}(t)$ the Heisenberg operator $e^{+(i / \hbar) H_{\mathrm{m}} t} \rho_{q} e^{-(i / \hbar) H_{\mathrm{m}} t}$, and the more compact notation $\Delta E_{q}(\boldsymbol{p})=E_{p+q}-E_{p}$ for the energy transfer has been used. Since the system is supposed to be homogeneous, the correlation function selects the contributions for which $\boldsymbol{q}=\boldsymbol{q}^{\prime}$, and exploiting the identity

$$
1=\int d t \delta\left(t-\left[\tau^{\prime}-\tau\right]\right)=\int d t \int \frac{d E}{2 \pi \hbar} \exp \left[(i / \hbar) E\left(t-\left[\tau^{\prime}-\tau\right]\right)\right]
$$

we have

$$
\begin{aligned}
\mathcal{L}(\hat{\varrho})= & \frac{2 \varepsilon}{\hbar} \sum_{p p^{\prime}} \sum_{q}^{\prime}|\tilde{t}(q)|^{2} e^{(i / \hbar) \boldsymbol{q} \cdot \hat{x}}|\boldsymbol{p}\rangle\left\langle\boldsymbol{p}|\hat{\varrho}| \boldsymbol{p}^{\prime}\right\rangle\left\langle\boldsymbol{p}^{\prime}\right| e^{-(i / \hbar) \boldsymbol{q} \cdot \hat{\boldsymbol{x}}} \frac{1}{\hbar^{2}} \int_{0}^{\infty} d \tau e^{-(\varepsilon / \hbar) \tau} \int_{0}^{\infty} d \tau^{\prime} e^{-(\varepsilon / \hbar) \tau^{\prime}} \int d E \\
& \times \exp \left\{-(i / \hbar)\left[\Delta E_{q}(\boldsymbol{p})-E\right] \tau\right\} \exp \left\{+(i / \hbar)\left[\Delta E_{q}\left(\boldsymbol{p}^{\prime}\right)-E\right] \tau^{\prime}\right\} \frac{1}{2 \pi \hbar} \int d t e^{(i / \hbar) E t}\left\langle\boldsymbol{\rho}_{q}^{\dagger} \rho_{q}(t)\right\rangle \\
& -\frac{\varepsilon}{\hbar} \sum_{p} \sum_{q}^{\prime}|\tilde{t}(q)|^{2}\{|\boldsymbol{p}\rangle\langle\boldsymbol{p}|, \hat{\varrho}\} \frac{1}{\hbar^{2}} \int_{0}^{\infty} d \tau e^{-(\varepsilon / \hbar) \tau} \int_{0}^{\infty} d \tau^{\prime} e^{-(\varepsilon / \hbar) \tau^{\prime}} \\
& \times \int d E \exp \left\{-i / \hbar\left[\Delta E_{q}(\boldsymbol{p})-E\right] \tau\right\} \exp \left\{+i / \hbar\left[\Delta E_{q}(\boldsymbol{p})-E\right] \tau^{\prime}\right\} \frac{1}{2 \pi \hbar} \int d t e^{(i / \hbar) E t}\left\langle\boldsymbol{\rho}_{q}^{\dagger} \boldsymbol{\rho}_{q}(t)\right\rangle .
\end{aligned}
$$

We can now meaningfully undo the Laplace transform, coming to

$$
\begin{aligned}
\mathcal{L}(\hat{\varrho})= & \frac{2 \varepsilon}{\hbar} \sum_{p p^{\prime}} \sum_{q}{ }^{\prime}|\widetilde{t}(q)|^{2} e^{(i / \hbar) \boldsymbol{q} \cdot \hat{x}}|\boldsymbol{p}\rangle\left\langle\boldsymbol{p}|\hat{\varrho}| \boldsymbol{p}^{\prime}\right\rangle\left\langle\boldsymbol{p}^{\prime}\right| e^{-(i / \hbar) \boldsymbol{q} \cdot \hat{\boldsymbol{x}}} \\
& \times \int d E \frac{\varepsilon}{\pi} \frac{1}{E-\Delta E_{q}(\boldsymbol{p})+i \varepsilon} \frac{1}{E-\Delta E_{q}\left(\boldsymbol{p}^{\prime}\right)-i \varepsilon} \frac{1}{2 \pi \hbar} \int d t e^{(i / \hbar) E t}\left\langle\boldsymbol{\rho}_{q}^{\dagger} \boldsymbol{\rho}_{q}(t)\right\rangle-\frac{\varepsilon}{\hbar} \sum_{p} \sum_{q}{ }^{\prime}|\tilde{t}(q)|^{2}\{|\boldsymbol{p}\rangle\langle\boldsymbol{p}|, \hat{\varrho}\} \\
& \times \int d E \frac{\varepsilon}{\pi} \frac{1}{E-\Delta E_{q}(\boldsymbol{p})-i \varepsilon} \frac{1}{E-\Delta E_{q}(\boldsymbol{p})+i \varepsilon} \frac{1}{2 \pi \hbar} \int d t e^{(i / \hbar) E t}\left\langle\boldsymbol{\rho}_{q}^{\dagger} \boldsymbol{\rho}_{q}(t)\right\rangle .
\end{aligned}
$$


If we now exploit the quasidiagonality of $\hat{\varrho}$, linked to its slow variability, thus substituting in the denominators of the first term $\boldsymbol{p}, \boldsymbol{p}^{\prime}$ with the symmetric expression $\frac{1}{2}\left(\boldsymbol{p}+\boldsymbol{p}^{\prime}\right)$, we obtain the expression

$$
\begin{aligned}
\mathcal{L}(\hat{\varrho})= & \frac{2 \pi}{\hbar} \sum_{p p^{\prime}} \sum_{q}{ }^{\prime}|\tilde{t}(q)|^{2} e^{(i / \hbar) \boldsymbol{q} \cdot \hat{x}}|\boldsymbol{p}\rangle\left\langle\boldsymbol{p}|\hat{\varrho}| \boldsymbol{p}^{\prime}\right\rangle\left\langle\boldsymbol{p}^{\prime}\right| e^{-(i / \hbar) \boldsymbol{q} \cdot \hat{\boldsymbol{x}}} \frac{1}{2 \pi \hbar} \int d t e^{(i / \hbar) \Delta E_{q}\left[\left(\boldsymbol{p}+\boldsymbol{p}^{\prime}\right) / 2\right] t}\left\langle\boldsymbol{\rho}_{q}^{\dagger} \boldsymbol{\rho}_{q}(t)\right\rangle \\
& -\frac{\pi}{\hbar} \sum_{p} \sum_{q}{ }^{\prime}|\tilde{t}(q)|^{2}\{|\boldsymbol{p}\rangle\langle\boldsymbol{p}|, \hat{\varrho}\} \frac{1}{2 \pi \hbar} \int d t e^{(i / \hbar) \Delta E_{q}(\boldsymbol{p}) t}\left\langle\boldsymbol{\rho}_{q}^{\dagger} \boldsymbol{\rho}_{q}(t)\right\rangle .
\end{aligned}
$$

The correlation functions appearing in Eq. (19) are exactly the dynamic structure factor multiplied by $N$ and evaluated for a momentum transfer $\boldsymbol{q}$ and energy transfers $\Delta E_{q}[(\boldsymbol{p}$ $\left.\left.+\boldsymbol{p}^{\prime}\right) / 2\right]$ and $\Delta E_{q}(\boldsymbol{p})$, respectively, as can be seen by comparison with Eq. (10). To see under which conditions the obtained master equation (16) takes a Lindblad structure, we consider an approximation of the form (12), which will generally depend on the smoothness of the energy dependence of the dynamic structure factor, but is actually less demanding than it might seem, since in the expression (19) one has to consider a sum over $\boldsymbol{p}$ and $\boldsymbol{p}^{\prime}$ with the matrix elements $\left\langle\boldsymbol{p}|\hat{\varrho}| \boldsymbol{p}^{\prime}\right\rangle$ of the statistical operator. In the continuum limit we therefore obtain the master equation

$$
\begin{aligned}
\frac{d \hat{\varrho}}{d t}= & -\frac{i}{\hbar}\left[\hat{H}_{0}, \hat{\varrho}\right]+\mathcal{L}(\hat{\varrho}) \\
= & -\frac{i}{\hbar}\left[\frac{\hat{\boldsymbol{p}}^{2}}{2 M}, \hat{\varrho}\right]+\frac{2 \pi}{\hbar}(2 \pi \hbar)^{3} n \int d^{3} \boldsymbol{q}|\widetilde{t}(q)|^{2} \\
& \times\left[e^{(i / \hbar) \boldsymbol{q} \cdot \hat{\boldsymbol{x}}} \sqrt{S(\boldsymbol{q}, \hat{\boldsymbol{p}})} \hat{\varrho} \sqrt{S(\boldsymbol{q}, \hat{\boldsymbol{p}})} e^{-(i / \hbar) \boldsymbol{q} \cdot \hat{\boldsymbol{x}}}\right. \\
& \left.-\frac{1}{2}\{S(\boldsymbol{q}, \hat{\boldsymbol{p}}), \hat{\varrho}\}\right],
\end{aligned}
$$

which still has the form (13), but is much more general since now the dynamic structure factor does not necessarily describe a free gas. This result allows for the extension of the usefulness of the master equation to cases in which the correlation function cannot be directly evaluated, but a suitable phenomenological model is available, e.g., determined in terms of scattering experiments.

\section{QUANTUM BROWNIAN MOTION AND QUANTUM STATISTICS}

We are now interested in the Brownian limit $\alpha=m / M$ $\ll 1$, considering the dynamics of a free particle interacting through collisions with a gas of much lighter particles. Having an expression valid for both a Fermi or Bose gas, it is particularly interesting to evaluate the correction brought about by quantum statistics to the typical models of quantum Brownian motion. In the limit $\alpha \ll 1$, expressions (6) and (11) become, respectively,

$$
\begin{aligned}
S_{\mathrm{B} / \mathrm{F}}(\boldsymbol{q}, \boldsymbol{p}, \alpha \ll 1)= & \mp \frac{1}{(2 \pi \hbar)^{3}} \frac{2 \pi m^{2}}{n \beta q} \frac{1}{1-e^{\beta\left[q^{2} / 2 M+\boldsymbol{q} \cdot \boldsymbol{p} / M\right]}} \\
& \times \log \left[\frac{1 \mp z e^{-(\beta / 8 m) q^{2}} e^{-(\beta / 2)\left[q^{2} / 2 M+\boldsymbol{q} \cdot \boldsymbol{p} / M\right]}}{\left.1 \mp z e^{-(\beta / 8 m) q^{2}} e^{+(\beta / 2)\left[q^{2} / 2 M+\boldsymbol{q} \cdot \boldsymbol{p} / M\right]}\right]}\right. \\
S_{\mathrm{MB}}(\boldsymbol{q}, \boldsymbol{p}, \alpha \ll 1)= & \frac{1}{(2 \pi \hbar)^{3}} \frac{2 \pi m^{2}}{n \beta q} z e^{-(\beta / 8 m) q^{2}} \\
& \times \exp \left\{-(\beta / 2)\left[\frac{q^{2}}{2 M}+\frac{\boldsymbol{q} \cdot \boldsymbol{p}}{M}\right]\right\}, \quad(22)
\end{aligned}
$$

or expressed in terms of momentum and energy transfer

$$
\begin{aligned}
S_{\mathrm{B} / \mathrm{F}}(q, E, \alpha \ll 1)= & \mp \frac{1}{(2 \pi \hbar)^{3}} \frac{2 \pi m^{2}}{n \beta q} \frac{1}{1-e^{\beta E}} \\
& \times \log \left[\frac{1 \mp z e^{-(\beta / 8 m) q^{2}} e^{-(\beta / 2) E}}{1 \mp z e^{-(\beta / 8 m) q^{2}} e^{+(\beta / 2) E}}\right] \\
S_{\mathrm{MB}}(q, E, \alpha \ll 1)= & \frac{1}{(2 \pi \hbar)^{3}} \frac{2 \pi m^{2}}{n \beta q} z e^{-(\beta / 8 m) q^{2}} e^{-(\beta / 2) E},
\end{aligned}
$$

still satisfying the principle of detailed balance [16]. In the Boltzmann case, as mentioned above, expression (22) exactly fulfills (12) and the generator in Eq. (15) takes the particularly simple form $L_{\mathrm{B} / \mathrm{F}}(\boldsymbol{q}, \hat{\boldsymbol{p}}, \hat{\boldsymbol{x}}) \propto e^{(i / \hbar) \boldsymbol{q} \cdot \hat{\boldsymbol{x}}} e^{-(\beta / 4 M) \boldsymbol{q} \cdot \hat{\boldsymbol{p}}}$, so that one obtains for an isotropic medium the master equation given in [7]

$$
\begin{aligned}
\frac{d \hat{\varrho}}{d t}= & -\frac{i}{\hbar}\left[\hat{H}_{0}, \hat{\varrho}\right]+z \frac{4 \pi^{2} m^{2}}{\beta \hbar} \int d^{3} \boldsymbol{q} \frac{|\widetilde{t}(q)|^{2}}{q} e^{-(\beta / 8 m) q^{2}} \\
& \times\left[e^{(i / \hbar) \boldsymbol{q} \cdot \hat{x}} e^{-(\beta / 4 M) \boldsymbol{q} \cdot \hat{p}} \hat{\varrho} e^{-(\beta / 4 M) \boldsymbol{q} \cdot \hat{p}} e^{-(i / \hbar) \boldsymbol{q} \cdot \hat{\boldsymbol{x}}}\right. \\
& \left.-\frac{1}{2}\left\{e^{-(\beta / 2 M) \boldsymbol{q} \cdot \hat{\boldsymbol{p}}}, \hat{\varrho}\right\}\right]
\end{aligned}
$$

To recover the equation describing quantum Brownian motion, one goes over to small momentum transfer, strongly favored by the kinematics of the collisions, considering terms up to second order in $\boldsymbol{q}$ or equivalently bilinear in $\hat{\boldsymbol{x}}$ and $\hat{\boldsymbol{p}}$, thus obtaining an equation in close analogy to the 
classical description, with a friction force proportional to velocity. The result for a Boltzmann gas is

$$
\begin{aligned}
\frac{d \hat{\varrho}}{d t}= & -\frac{i}{\hbar}\left[\hat{\boldsymbol{H}}_{0}, \hat{\varrho}\right]-z \sum_{i=1}^{3}\left\{\frac{D_{p p}}{\hbar^{2}}\left[\hat{\boldsymbol{x}}_{i},\left[\hat{\boldsymbol{x}}_{i}, \hat{\varrho}\right]\right]\right. \\
& \left.+\frac{D_{x x}}{\hbar^{2}}\left[\hat{\boldsymbol{p}}_{i},\left[\hat{\boldsymbol{p}}_{i}, \hat{\varrho}\right]\right]+\frac{i}{\hbar} \gamma\left[\hat{\boldsymbol{x}}_{i},\left\{\hat{\boldsymbol{p}}_{i}, \hat{\varrho}\right\}\right]\right\}
\end{aligned}
$$

with

$$
\begin{gathered}
D_{p p}=\frac{2}{3} \frac{\pi^{2} m^{2}}{\beta \hbar} \int d^{3} \boldsymbol{q}|\tilde{t}(q)|^{2} q e^{-(\beta / 8 m) q^{2},} \\
D_{x x}=(\beta \hbar / 4 M)^{2} D_{p p}, \quad \gamma=(\beta / 2 M) D_{p p},
\end{gathered}
$$

and has the particular feature that it can be written in Lindblad form in terms of a single generator [7,19]. Starting from Eq. (21) one can perform the same limit of small momentum transfer corresponding through the physical interpretation of the dynamic structure factor to the macroscopic, longwavelength properties of the system, thus calculating the correction due to quantum statistics to the master equation describing quantum Brownian motion. To do this one considers the Taylor expansion of the logarithms in Eq. (21), leading to the following compact expression as a power series in the fugacity $z$

$$
\begin{aligned}
S_{\mathrm{B} / \mathrm{F}}(q, E, \alpha \ll 1) & \\
= & S_{\mathrm{MB}}(q, E, \alpha \ll 1) \\
& \times\left[1+\sum_{k=1}^{\infty}( \pm)^{k} \frac{z^{k}}{k+1} e^{-k(\beta / 8 m) q^{2}} e^{-k(\beta / 2) E} \sum_{n=0}^{k} e^{n \beta E}\right],
\end{aligned}
$$

which has to be substituted in Eq. (13), keeping terms up to second order in $q$. The result one obtains is actually remarkably simple; the operator structure is not changed, nor the simple generator feature, but the fugacity appears through the expression $z /(1 \mp z)$ rather than linearly. For a Bose or Fermi gas at finite temperature one has

$$
\begin{aligned}
\frac{d \hat{\varrho}}{d t}= & -\frac{i}{\hbar}\left[\hat{H}_{0}, \hat{\varrho}\right]-\frac{z}{1 \mp z} \sum_{i=1}^{3}\left\{\frac{D_{p p}}{\hbar^{2}}\left[\hat{\boldsymbol{x}}_{i},\left[\hat{\boldsymbol{x}}_{i}, \hat{\varrho}\right]\right]\right. \\
& \left.+\frac{D_{x x}}{\hbar^{2}}\left[\hat{\boldsymbol{p}}_{i},\left[\hat{\boldsymbol{p}}_{i}, \hat{\varrho}\right]\right]+\frac{i}{\hbar} \gamma\left[\hat{\boldsymbol{x}}_{i},\left\{\hat{\boldsymbol{p}}_{i}, \hat{\varrho}\right\}\right]\right\},
\end{aligned}
$$

where the coefficient $z /(1 \mp z)$ at finite temperature is actually well defined because $z$ is in the range $0 \leqslant z<1$ for Bose particles and positive for Fermi particles.

Equation (27), expressing the correction due to quantum statistics in the equation describing quantum Brownian motion, together with Eq. (20), giving a completely positive time evolution for a particle interacting with a macroscopic system at equilibrium in terms of a momentum displacement operator and the dynamic structure factor of the system, are the main results of this paper. Comparing Eqs. (27) with (24) one sees that the friction coefficient given in the Boltzmann case by

$$
\gamma_{\mathrm{MB}}=z \frac{\beta}{2 M} D_{p p}=z \frac{1}{3} \frac{\pi^{2} m^{2}}{M \hbar} \int d^{3} \boldsymbol{q}|\tilde{t}(q)|^{2} q e^{-(\beta / 8 m) q^{2}}
$$

is now substituted by

$$
\gamma_{\mathrm{B} / \mathrm{F}}=\frac{\gamma_{\mathrm{MB}}}{1 \mp z},
$$

enhanced or suppressed according to statistics. Both Eqs. (24) and (27) retain the property of complete positivity satisfied by Eq. (14), are invariant under translation and rotation, and admit a stationary solution of the canonical form $\hat{\varrho} \propto e^{-\beta\left(\hat{p}^{2} / 2 M\right)}$. The single generator feature is due to the fact that the coefficients satisfy the relationship $D_{p p} D_{x x}$ $=\hbar^{2} \gamma^{2} / 4$.

\section{SUMMARY AND OUTLOOK}

We have considered the problem of the motion of a test particle interacting through collisions with a fluid, following the approach outlined in Refs. [14,20,21], which has already been successfully applied to the case of neutron optics [22]. The microscopic derivation allows some insights into the conditions under which a master equation of the Lindblad type, driving a completely positive time evolution, can be obtained, thus giving a concrete physical example contributing to the debate on the relevance of complete positivity [13]. Provided the statistical operator is sufficiently diagonal in momentum representation with respect to the energy dependence of the dynamic structure factor, the master equation (20) is obtained, where only quantities of physical interest appear; the scattering cross section for the single twobody collisions, given by the square of the $T$ matrix; the generator of translations in momentum space, and the dynamic structure factor, keeping the statistical properties of the medium into account, combined through the expression $L(\boldsymbol{q}, \hat{\boldsymbol{p}}, \hat{\boldsymbol{x}})=e^{(i / \hbar) \boldsymbol{q} \cdot \hat{\boldsymbol{x}}} \sqrt{S(\boldsymbol{q}, \hat{\boldsymbol{p}})}$. This structure is remarkably simple and describes a dynamics in which the motion of the test particle is linked through this particular two-point correlation function to the spectrum of spontaneous fluctuations of the system. Starting from this general structure and explicitly calculating the dynamic structure factor for the case of a free gas, one can consider the particularly relevant case of Brownian motion, when the test particle is much heavier than the particles making up the gas. In the case of a Boltzmann gas one recovers, for small momentum transfer, a typical structure of generator of quantum Brownian motion, given by Eq. (24), in which all coefficients are determined and the dissipative part of the generator depends linearly on the fugacity. The case of a quantum gas is also considered, and in this case the generator has the structure (27), with the 
dissipative part depending on the fugacity $z$ through the expression $z /(1 \mp z)$, thus giving the connection (29) between the friction coefficients in the different cases.

We hope that this fundamental study on the general features of a master equation describing the motion of a test particle in a gas, putting in major evidence the dynamic structure factor and showing the relationship between this structure and the equation, analogous to the Fokker-Planck equation, describing quantum Brownian motion, could be a sound starting point for future extensions and applications, especially in connection with degenerate regimes at very low temperatures, where the dynamic structure factor is now be- ing intensively studied both at an experimental [23] and theoretical level [24].

\section{ACKNOWLEDGMENTS}

The author would like to thank Professor L. Lanz for many useful suggestions and careful reading of the manuscript and Professor A. Barchielli for useful discussions. This work was partially supported by the Alexander von Humboldt-Stiftung and by MURST under Cofinanziamento and Progetto Giovani.
[1] D. Giulini et al., Decoherence and the Appearance of a Classical World in Quantum Theory (Springer-Verlag, Berlin, 1996).

[2] M. Brune et al., Phys. Rev. Lett. 77, 4887 (1996); C.J. Myatt et al., Nature (London) 403, 269 (2000).

[3] M. Anderson et al., Science 269, 198 (1995); F. Dalfovo et al., Rev. Mod. Phys. 71, 463 (1999); G. M. Tino and M. Inguscio, Riv. Nuovo Cimento 22, 1 (1999).

[4] H. Spohn, Rev. Mod. Phys. 53, 569 (1980).

[5] B. DeMarco and D.S. Jin, Science 285, 1703 (1999).

[6] M. Yamashita, M. Koashi, and N. Imoto, Phys. Rev. A 2243, 59 (1999); G. Ferrari, ibid. 59, R4125 (1999); C.W. Gardiner and P. Zoller, ibid. 55, 2902 (1997).

[7] B. Vacchini, Phys. Rev. Lett. 84, 1374 (2000).

[8] R. F. O'Connell, Phys. Rev. Lett. (to be published); B. Vacchini, Phys. Rev. Lett. (to be published).

[9] G. Lindblad, Rep. Math. Phys. 10, 393 (1976); A. Barchielli, Nuovo Cimento B 74, 113 (1983); G. Lindblad, J. Math. Phys. 39, 2763 (1998).

[10] G. Lindblad, Commun. Math. Phys. 48, 119 (1976); K. Kraus, Lecture Notes in Physics Vol. 190 (Springer-Verlag, Berlin, 1983); K.-E. Hellwig, Int. J. Theor. Phys. 34, 1467 (1995); R. Alicki and K. Lendi, Lecture Notes in Physics Vol. 286 (Springer-Verlag, Berlin, 1987).

[11] A. Săndulescu and H. Scutaru, Ann. Phys. (N.Y.) 173, 277 (1987); A. Isar, Fortschr. Phys. 47, 855 (1999).
[12] V. Ambegaokar, Ber. Bunsenges. Phys. Chem. 95, 400 (1991); P. Pechukas, in Large Scale Molecular Systems, edited by W. Gans, A. Blumen, and A. Amann, NATO ASI Series (Plenum, New York, 1991), Vol. 258, p. 123; A. Isar, A. Sandulescu, and W. Scheid, J. Math. Phys. 34, 3887 (1993); D. Kohen, C.C. Marston, and D.J. Tannor, J. Chem. Phys. 107, 5236 (1997).

[13] P. Pechukas, Phys. Rev. Lett. 73, 1060 (1994); 75, 3021 (1995); R. Alicki, ibid. 75, 3020 (1995); R.C. de Berrêdo et al., Phys. Scr. 57, 533 (1998).

[14] L. Lanz and B. Vacchini, Int. J. Theor. Phys. 36, 67 (1997).

[15] L. van Hove, Phys. Rev. 95, 249 (1954).

[16] S. W. Lovesey, Theory of Neutron Scattering from Condensed Matter (Clarendon, Oxford, 1984).

[17] H. R. Glyde, Excitations in Solid and Liquid Helium (Clarendon, Oxford, 1994).

[18] B. Vacchini (unpublished).

[19] B. Vacchini, Z. Naturforsch 56a, 230 (2001).

[20] L. Lanz and B. Vacchini, Int. J. Theor. Phys. 37, 545 (1998).

[21] L. Lanz, O. Melsheimer, and B. Vacchini, in Quantum Communication, Computing, and Measurement, edited by $\mathrm{O}$. Hirota, A. S. Holevo, and C. M. Caves (Plenum, New York, 1997), p. 339.

[22] L. Lanz and B. Vacchini, Phys. Rev. A 56, 4826 (1997).

[23] J. Stenger et al., Phys. Rev. Lett. 82, 4569 (1999); D.M. Stamper-Kurn et al., ibid. 83, 2876 (1999).

[24] F. Zambelli et al., Phys. Rev. A 61, 063608 (2000). 